\title{
Multifunctional actuation systems responding to chemical gradients
}

\section{Citation}

Zarzar, Lauren D., Qihan Liu, Ximin He, Yuhang Hu, Zhigang Suo, and Joanna Aizenberg. 2012. Multifunctional Actuation Systems Responding to Chemical Gradients. Soft Matter 8, no, 32: 8289. doi:10.1039/c2sm26064f.

\section{Published Version}

doi:10.1039/C2SM26064F

\section{Permanent link}

http://nrs.harvard.edu/urn-3:HUL.InstRepos:27662701

\section{Terms of Use}

This article was downloaded from Harvard University's DASH repository, and is made available under the terms and conditions applicable to Open Access Policy Articles, as set forth at http:// nrs.harvard.edu/urn-3:HUL.InstRepos:dash.current.terms-of-use\#OAP

\section{Share Your Story}

The Harvard community has made this article openly available.

Please share how this access benefits you. Submit a story.

Accessibility 


\title{
Multifunctional Actuation Systems Responding to Chemical Gradients
}

\author{
Lauren D. Zarzar, ${ }^{a}$ Qihan Liu, ${ }^{b}$ Ximin He,${ }^{b, c}$ Yuhang Hu, ${ }^{b}$ Zhigang Suo, ${ }^{b}$ and Joanna Aizenberg ${ }^{a, b, c}$ \\ Received (in $X X X, X X X)$ Xth $X X X X X X X X X 20 X X$, Accepted Xth XXXXXXXXX 20XX \\ DOI: $10.1039 / b 000000 x$
}

\begin{abstract}
5 The ability to manipulate the movement of surface microstructures is essential for the development of dynamic, responsive materials. We demonstrate that in addition to bulk actuation upon drying, a unique type of highly localized, directional actuation can be achieved when microstructures

10 embedded in $\mathbf{p H}$-responsive gel are exposed to $\mathbf{p H}$-gradients. Theory and modelling elucidates the underlying mechanism behind this novel approach to inducing responsive actuation.
\end{abstract}

Control over the dynamic movement of nano/microstructures is fundamentally important for actuators and sensors, tunable optics, 15 micromanipulation, and the design of "smart" responsive surfaces. ${ }^{1,2}$ Programming the location of response, the direction of movement, and the extent of structural reconfiguration on the microscale are all important characteristics of tunable, dynamic materials. Natural systems have mastered the ability to use 20 chemical cues, often applied not as a uniform stimulus, but in the form of chemical or electrochemical gradients, to direct the motion of structures from the nanoscale up. For example, the beating of flagella is driven by proton motive force, ${ }^{3}$ and the direction of movement of bacteria can be guided by chemotaxis. ${ }^{4}$

25 The ability of a system to respond to a gradient chemical stimulus rather than a homogeneous environment is accompanied by an inherent directionality, allowing for the chemical stimulus to trigger not only the location of response, but also the direction of movement along the gradient.

30 Although the versatility, tunability, and controllability of artificial responsive actuators would benefit from such directional sensitivity, few systems take advantage of chemical gradients or interfaces to direct the actuation of materials. For instance, chemical gradients have been used to generate the oriented 35 movement of metallic nanorods, similar in concept to chemotaxis. ${ }^{5}$ Recently, there has been significant interest in responsive polymers, such as hydrogels, for chemo-responsive actuators due to their reversible and highly tunable volume change as well as sensitivity to a wide range of stimuli including 40 humidity, temperature, $\mathrm{pH}$, light, and redox state. ${ }^{6,7}$ Although typically such hydrogels are used and studied in regards to their response to a homogeneous stimulus (i.e. the polymer network is either swollen or contracted in a certain state), introducing localized stimuli or gradients of stimuli across a gel can be 45 advantageous for a number of purposes. Cargo transport, for example, was accomplished using gels that swell and shrink in response to oscillations of the Belousov-Zhabotinsky reaction, transporting particles along with the moving interface between swollen and contracted gel regions. ${ }^{8}$ Further exploration of how
50 chemical interfaces and gradients can be used to manipulate hydrogels and induce reversible system directionality is therefore of fundamental interest.

Combination of a chemically responsive material, such as hydrogel, with structures which can be reconfigured and provide ${ }_{55}$ the composite system with unique structural properties is a powerful concept that we have recently been exploring through the use of hydrogels as a "muscle" to dynamically actuate gelembedded high-aspect-ratio microstructures. ${ }^{9}$ Patterned gel thickness $^{10}$ and asymmetry in the structure geometry itself ${ }^{11}$

${ }_{60}$ provide some control over the bending direction of structures within such systems. However, in hybrid systems previously reported $^{10-14}$ the structural actuation responds to homogenous stimuli, and the cycling of gel expansion and contraction results in actuation between pre-programmed configurations inherent to ${ }_{65}$ the physical makeup of the surface. To expand the versatility and generate a system that is responsive to a far greater range of stimuli scenarios, both in terms of spatial and directional variations, we aimed to use gradient chemical signals to trigger actuation between numerous configurations. Here, we develop a

70 multifunctional actuation system in which microstructures embedded in a $\mathrm{pH}$-responsive gel can undergo multiple actuation scenarios: (i) highly localized, directional movement of embedded microstructures in response to $\mathrm{pH}$ gradients, but not to the change in the bulk $\mathrm{pH}$, as in previously described $\mathrm{pH}$ ${ }_{75}$ responsive systems, ${ }^{11}$ (ii) uniform large-area actuation upon hydration/dehydration. We explore the fundamental mechanisms of such novel behavior including investigation of how the chemical gradient is translated to the physical structure of the hydrogel and in turn how the system provides the ability to 80 control the direction of bending, location and area of bending, and degree of bending of embedded structures.

The hybrid surfaces used here consist of high-aspect-ratio flexible microstructures embedded in a responsive hydrogel, where the volume change of the hydrogel can drive the 85 movement, or actuation, of the structures. The hydrogel is $\mathrm{pH}-$ responsive poly(acrylamide-co-acrylic acid), which has a volume phase transition at $\mathrm{pH} \approx 4-5$ corresponding to the $\mathrm{pK}_{\mathrm{a}}$ of acrylic acid (4.3), with embedded structures made from glycidyl methacrylate-modified epoxy. To fabricate a hybrid surface, 90 hydrogel precursor solution was placed on the structured surface and allowed to spread under confinement of a glass coverslip. The hydrogel was crosslinked by UV-initiated polymerization in this confined state. ${ }^{11}$ Since the poly(acrylamide-co-acrylic acid) hydrogel precursor solution is quite acidic $(\mathrm{pH} \approx 2)$, the hydrogel 95 is polymerized in a contracted state. 
a) 3D Schematic
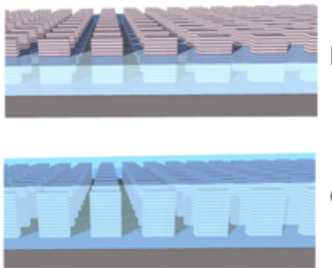

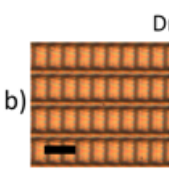

Dry gel

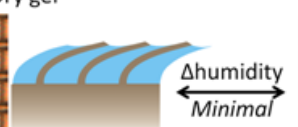

c)

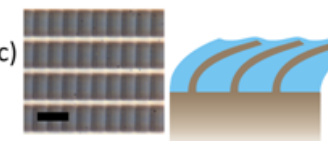

actuation
Contracted gel, submerged

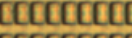

(1)

CEDIIII

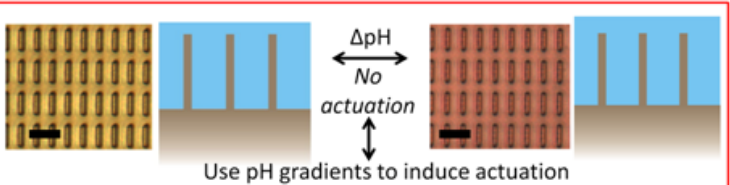

Fig. 1 Tuning actuation response with humidity and $\mathrm{pH}$. a) Schematic 3-D representation of an array of microfin structures embedded in hydrogel. bc) Each panel contains a top-view optical image along with a schematic side-view cross-section. The coloration in the optical images arises from bromophenol blue $\mathrm{pH}$ indicator added to the solution. b) For surfaces in which structures are bent in a contracted gel in acidic conditions (middle), we observe pH-responsive actuation (between middle and right), but minimal actuation between the contracted, acidic state and dry state (middle and left). c) For surfaces in which structures are upright in the contracted gel in acidic conditions (middle), we do not observe pH-responsive actuation (between middle and right), but we can still observe humidity-responsive actuation (between middle and left). The red box highlights the conditions under which we used $\mathrm{pH}$ gradients to actuate structures. Scale bars $=10 \mu \mathrm{m}$.

By controlling the initial position of the structures (bent versus upright) with respect to the initial state of the hydrogel (contracted versus swollen), we could generate a system that will either actuate or remain static in response to the bulk $\mathrm{pH}$ change 5 (Figure 1). In particular, we anticipated that if the structures are bent in the initial state of the contracted submerged hydrogel, the structures would actuate into an upright orientation upon the increase in the bulk $\mathrm{pH}$ whereas if the structures are oriented upright in the contracted submerged gel, further gel expansion 10 upon $\mathrm{pH}$ increase would not change their orientation. The latter system should, however, show significant actuation upon drying. Indeed, when we generated structures that were bent after the polymerization process as previously described (Figure $1 \mathrm{~b}$, middle), ${ }^{11}$ we observed homogeneous $\mathrm{pH}$-responsive actuation 15 because structures could be pushed to the upright position by the hydrogel swelling in basic conditions (Figure 1b, right). Upon drying, the original bent structures show only minimal actuation further down to the surface due to the additional contraction of the gel (Figure 1b, left). When we fabricated surfaces in which 20 the structures in their original state remained upright after hydrogel polymerization by tailoring the initial fabrication and confinement conditions (Figure 1c, middle) (Supporting Information), we observed vertical expansion of the gel, but no actuation of the microstructures upon increase of $\mathrm{pH}$ (Figure 1c,

${ }_{25}$ right). In this case, however, significant actuation of the microstructures takes place upon dehydration of the gel between the submerged state and the dry state (Figure 1c, left), as further gel shrinkage upon drying induced structures to buckle. ${ }^{15}$ In effect, we were able to shift the homogeneous actuation response 30 from $\mathrm{pH}$ to humidity by controlling the initial conditions of the system.

The absence of actuation in response to bulk $\mathrm{pH}$ made this particular case an ideal test bed for studying the actuation of structures solely in response to $\mathrm{pH}$ gradients. We incorporated 35 hybrid surfaces with $\mathrm{pH}$-sensitive hydrogel, but not responsive to homogeneous $\mathrm{pH}$ (Figure 1, red box), into microchannels (Figure 2a). Hydrogel was cured only in the area of the microchannel using a photomask, and the microchannel itself was made with double sided adhesive sealed at the top with a layer of 40 polydimethylsiloxane (PDMS). Aqueous hydrochloric acid solution $(\mathrm{pH}=2)$ and sodium hydroxide solution $(\mathrm{pH}=10)$ were pumped into the channel creating a sharp $\mathrm{pH}$ gradient at the laminar flow interface (Figure $2 b$ ). We investigated the actuation of both microfins (width $=1.2 \mu \mathrm{m}$, height $=11.5 \mu \mathrm{m}$, length $=10$ ${ }_{45} \mu \mathrm{m}$, side to side spacing $=5 \mu \mathrm{m}$, front to back spacing $=5 \mu \mathrm{m}$ ) and posts $($ diameter $=1.5 \mu \mathrm{m}$, height $=10 \mu \mathrm{m}$, pitch $=8 \mu \mathrm{m})$ and observed that structures always bent toward the direction of the acid within a "transition zone" of finite width across the interface while preserving an upright position farther from the interface 50 both in the acidic and basic channels (Figure 2c,d). We could reversibly control the bending direction of the structures and the position of the transition zone by changing the location, shape (Figure S1), and gradient direction; for example, as seen in Figure $2 \mathrm{c}$, structures bent in opposing directions simply by reversing the 55 direction of the $\mathrm{pH}$ gradient.

Since the gel at this $\mathrm{pH}$ interface was able to significantly shear and bend structures in the direction perpendicular to the acid/base interface, we anticipated that this force, when applied across different axes of an asymmetric embedded structure such as a 60 microfin, may also facilitate control over the degree to which structures were bent over. We therefore rotated the hydrogelembedded microfins within the microfluidic channel so that the $\mathrm{pH}$ interface was positioned at different angles relative to the embedded structures. To approximately calculate the degree of 65 structure bending, we assumed that the structures were bent from the substrate base and were not curved. When the lengths of the structures were oriented parallel to the $\mathrm{pH}$ interface (such as in Figure 2c), structures bent to angles of $30-35^{\circ}$; with an interface at a $60^{\circ}$ angle, structures' bending angle decreased to $20-25^{\circ}$, and 70 with the interface at an $80^{\circ}$ angle, structures bent only $10-15^{\circ}$ (Figure 2e). Furthermore, we observed that even surfaces that did actuate in response to homogeneous $\mathrm{pH}$ stimulus (such as those shown in Figure 1b) could still be affected by the $\mathrm{pH}$ gradient and induce structures to bend in the opposite direction to what would 75 be normally expected, but only over short distances (Figure 2f). Of course, such backward-bending behavior only took place where the force generated by the $\mathrm{pH}$ gradient opposed the buckling-induced actuation direction; in Figure 2f, we saw that when structures already preferentially bent in the direction facing 80 the acid, the actuation pattern was undisturbed, but backwardbending was induced when the preferred bending direction was toward the base.

The $\mathrm{pH}$ gradients used here were quite sharp and spanned only a few microns, but we observed the areas over which the 85 structures bent (the transition zone) to be significantly wider than the $\mathrm{pH}$ gradient itself. We hypothesized that this discrepancy may be due to the fact that the gel cannot immediately conform to the chemical signal due to physical constraints. Thicker gels would then require larger distances to reach their equilibrium swollen 90 and contracted states on either side of the interface than a thinner gel. To test the correlation of gel thickness with transition zone width, we created a surface with increasing hydrogel thickness along the length of the channel. As pictured in Figure $2 \mathrm{~g}$, we observed that the width of the transition zone indeed increased ${ }_{95}$ along with hydrogel thickness even while under the influence of the same $\mathrm{pH}$ interface. 
We used confocal microscopy to quantify this influence of the hydrogel thickness on structure actuation and investigate the hydrogel profile at the $\mathrm{pH}$ interface. To visualize both the actuation of the microfins and the topography of the hydrogel at 5 the same time, epoxy microfins were fluorescently dyed with pyrromethene 546 and $1 \mu \mathrm{m}$ red fluorescent microspheres were adhered to the surface of the hydrogel. Overlaying a confocal Zstack cross-section showing both the fluorescent microfins and gel-adhered microspheres with a bright field optical image to

10 clearly show the extent of structure actuation (Figure $3 \mathrm{a}$ ) revealed that structure bending in the transition zone correlated to the region over which the thickness profile of the gel was sloped. We could tune the transition zone from widths of $<50 \mu \mathrm{m}$ (less than 10 consecutively bending structures) to nearly $500 \mu \mathrm{m}(\sim 100$ 15 consecutively bending structures) simply by changing hydrogel thickness (Figure 3b).

To gain insight into the fundamental mechanism of how the $\mathrm{pH}$ gradient at the acid/base interface actually causes the bending of the structures, we modeled the surface deformation using a 20 nonlinear field theory of hydrogels which was implemented as a user subroutine in the commercial software ABAQUS. ${ }^{16}$ The crosslink density of the hydrogel was chosen such that the ratio of the shear modulus of the gel in acidic conditions over that of the epoxy matched the experimentally measured value

${ }_{25}$ GgelGepoxy $=0.37 \times 10-3$. Since the behavior of $\mathrm{pH}$-responsive gels can be also understood in terms of the osmotic pressure, ${ }^{17}$ we simulated the changes in the $\mathrm{pH}$ by prescribing a corresponding change in the osmotic pressure. Under homogeneous $\mathrm{pH}$ conditions in which structures are programmed to stand upright in 30 the contracted gel state (Figure 3c top), upon homogenous swelling the gel deforms vertically and increases in thickness; the gel is surface-attached and cannot expand laterally (Figure 3c bottom) and as a result structures do not actuate. However, under conditions of a sharp $\mathrm{pH}$ gradient (Figure $3 \mathrm{c}$ middle) we observed

35 that the hydrogel was additionally able to deform horizontally, and this displacement was always in the direction of the acidic region causing structures to be sheared toward the contracted gel. The calculated result is shown in Figure 3c, middle, where the gel color indicates the magnitude of horizontal displacement 40 leftward. Our model suggests that the largest horizontal displacement should take place near the acid/base interface and the hydrogel should gradually revert to zero horizontal displacement farther from the interface. This prediction correlates well with the experimental observation of structural ${ }_{45}$ bending within a transition zone of finite width. Furthermore, if influence from the embedded structures is neglected, the width of this transition zone should be proportional to the thickness of the gel, which is the only relevant length scale in the system. The experimentally determined correlation between the thickness of ${ }_{50}$ the gel and the width of the transition zone appeared to initially follow this predicted linear behavior (Figure 3b). At larger gel thicknesses $(>50 \mu \mathrm{m})$, however, we began to observe that the gel formed creases both along and perpendicular to the interface, and the linear relationship was no longer valid.

55 In summary, we have presented here a novel mechanism for gradient-responsive, hydrogel-driven actuation of microstructures. First, we effectively removed the homogeneous $\mathrm{pH}$ response of the hybrid systems made of $\mathrm{pH}$-responsive hydrogel, allowing us to explore the use of gradient $\mathrm{pH}$ stimuli to 60 affect tunable actuation via locally generated asymmetries in the hydrogel. We have demonstrated that by using $\mathrm{pH}$ gradient stimuli we can achieve a higher level of control over the actuation location, degree of structure reconfiguration, actuation direction, and general customizability and adaptiveness of the responsive ${ }_{65}$ hybrid surfaces. Furthermore, such surfaces now have a built-in
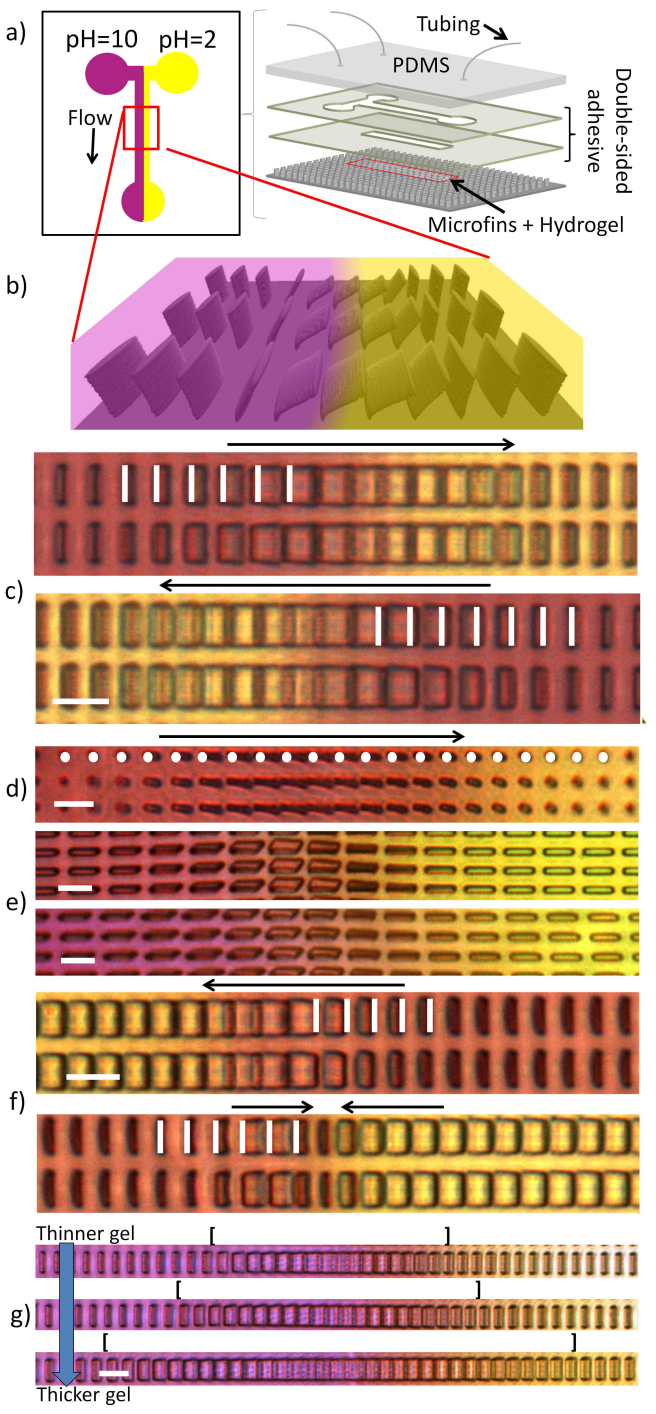

Fig. 2 Patterned actuation of microstructures driven by a pH-responsive hydrogel induced by a $\mathrm{pH}$ gradient, which was created by flowing acid and base in a microfluidic channel. a) Schematic representation of experimental setup. b) 3-D schematic showing an orientation of microfins within such a microfluidic channel, as demonstrated in (c). cg) Optical microscope images of actuated microstructures taken normal to the surface, showing that the direction of microstructure bending is always towards the acid. White lines or dots are place markers for the base of the structures to help visualize bending directions; bending directions are also marked with arrows above the micrographs. Color in all micrographs arises from $\mathrm{pH}$ indicator, bromophenol blue. Scale bars $=10 \mu \mathrm{m}$ : c) Bending direction can be switched by $\mathrm{pH}$ interface reversal: microfins are bending to the right when the acid is flowed on the right side of the channel (top), and the same fins are bending to the left when the acid is flowed on the left side of the same channel (bottom). d) Microposts showing the same behavior as the microfins and bending toward lower pH. e) Microfins like those in (c) but placed at different angles relative to the laminar flow interface exhibiting different bending angles. f) When a sample, which actuates in response to homogeneous $\mathrm{pH}$ as shown in Figure $1 \mathrm{~b}$, is put in a $\mathrm{pH}$ gradient, it exhibits either a uniform bending direction corresponding to the bending direction of the original structures (e.g. to the left in the top image, when the acid is flowed to the left), or unique backwardbending at the base/acid interface, when the acid is flowed against the original bending direction, to the right. g) A surface in which there is gradient hydrogel thickness; as the gel thickness increases, the width of the region in which structures bend (indicated by brackets above the micrographs) also increases given the same $\mathrm{pH}$ interface. 

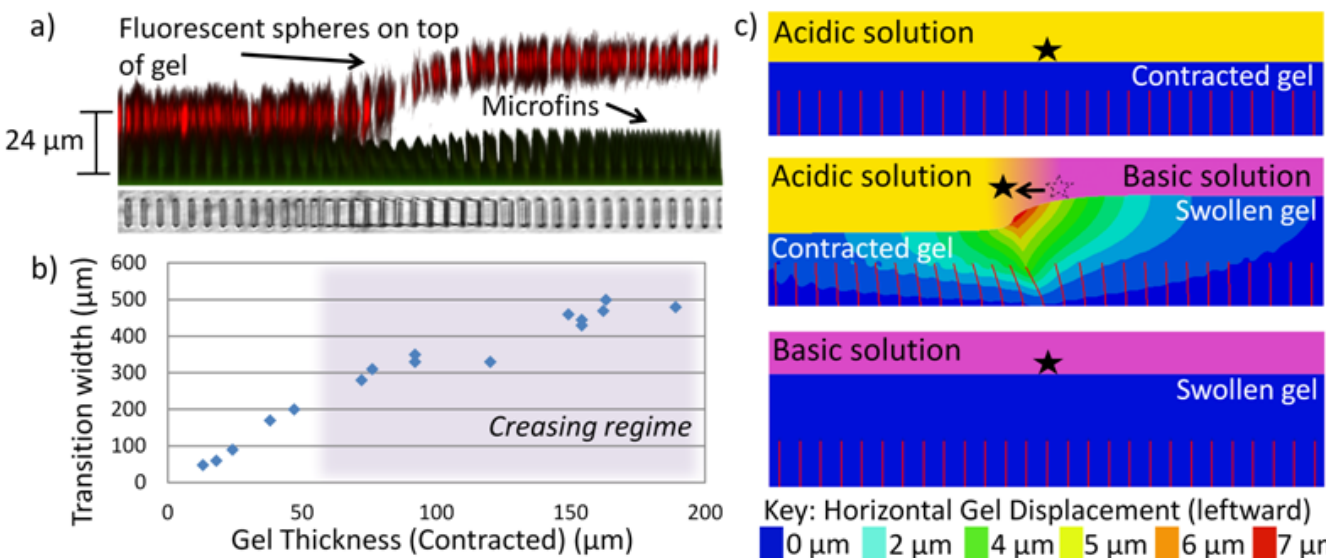

Key: Horizontal Gel Displacement (leftward)

$0 \mu \mathrm{m} \quad 2 \mu \mathrm{m} \quad 4 \mu \mathrm{m} \quad 5 \mu \mathrm{m} \quad 6 \mu \mathrm{m} \quad 7 \mu \mathrm{m}$

Fig. 3 Quantitative investigation of the mechanism of actuation and effect of the $\mathrm{pH}$ gradient on hydrogel thickness and profile shape. a) Exemplary confocal Z-stack cross-section of the microstructure/hydrogel surface while in a microfluidic channel under acid/base laminar flow. Red corresponds to $1 \mu \mathrm{m}$ red fluorescent particles adhered to the hydrogel surface and green corresponds to the microfins. Top-view optical image of the same area is aligned underneath to better visualize structure bending. b) Correlation of the transition width with hydrogel thickness (measured in contracted state) indicates a linear relationship for gels $<50 \mu \mathrm{m}$ thick before creasing in the gel occurs and the linear relationship ceases to hold. c) Finite element method (FEM) simulation of the hydrogel and embedded structures in contracted gel (top), across a sharp acid/base interface (middle) and in swollen gel (bottom). The star marks the center of the gel to emphasize the lateral shifting of the hydrogel in the $\mathrm{pH}$ gradient case (middle), which is the underlying mechanism for actuation. Gel color indicates the magnitude of leftward displacement. Background color indicates $\mathrm{pH}$ (yellow is acidic and purple is basic, correlating with the colors of the $\mathrm{pH}$ indicator used in Figures 1 and 2)

actuation response to humidity, providing extended combinatorial sensitivity to multiple stimuli, each with its characteristic actuation pattern. In particular, microstructures embedded in a thick layer of $\mathrm{pH}$-responsive gel similar to the ones in Figure 1c 5 would show either uniform actuation of all microstructures upon dehydration, or a highly localized stripe of actuated microstructures in response to a $\mathrm{pH}$ gradient, or no actuation in response to bulk $\mathrm{pH}$ change.

Through modeling and simulations we have been able to 10 explain the fundamental mechanism of this new method of hydrogel-driven actuation; lateral displacement of hydrogel occurring at the acid/base interface shears structures always in the direction of this displacement (towards contracted gel) providing the system with highly directional chemical sensitivity. We 15 expect that this new actuation mechanism may prove useful for precise reconfiguration of surface structures, especially for tunable optical systems, or as visual sensors which provide insight into gel dynamics and creasing studies. Furthermore, the mechanism of actuation which we describe here is highly general 20 and not only pH-specific, meaning that this method could be applicable for a range of environmentally-responsive hydrogels. Broadly, the multifunctional dynamic systems developed in this study, in particular the use of non-uniform chemical stimuli within environmentally-sensitive gels to induce structural 25 asymmetry and highly localized reconfiguration (in this case, the use of a $\mathrm{pH}$ gradient), coupled with the ability to induce uniform bulk actuation upon application of a different stimulus (in this case, the use of hydration/dehydration), may have far reaching impact on the growing field of "smart", adaptive and multi30 responsive systems.

\section{Acknowledgments}

We thank Dr. P. Kim, Dr. U. Salman, and Dr. A. Grinthal for discussions and Dr. A. Taylor and Dr. M. Khan for substrate fabrication. This work was supported by the DOE under award

${ }_{35}$ DE-SC0005247. L. D. Z. thanks the DoD for support through the NDSEG Fellowship Program as well as the NSF for support through the Graduate Research Fellowship Program

\section{Notes and references}

a Department of Chemistry and Chemical Biology, Harvard University, ${ }_{40}$ Cambridge, MA 02138, USA

${ }^{b}$ School of Engineering and Applied Sciences, Harvard University, Cambridge, MA 02138, USA

${ }^{c}$ Wyss Institute for Biologically Inspired Engineering, Harvard University, Cambridge, MA 02138, USA

45 EE-mail: jaiz@seas.harvard.edu

$\dagger$ Electronic Supplementary Information (ESI) available: includes details regarding fabrication and characterization. See DOI: 10.1039/b000000x/

1. S. A. Wilson, et. al , Mat. Sci. Eng. R-Reports, 2007, 56, 1.

50 2. Z.-g. Zhou and Z.-w. Liu, J. Bionic Eng., 2008, 5, 358.

3. M. D. Manson, P. Tedesco, H. C. Berg, F. M. Harold and C. Van Der Drift, P. Natl. Acad. Sci. USA, 1977, 74, 3060.

4. U. Alon, M. G. Surette, N. Barkai and S. Leibler, Nature, 1999, 397, 168.

55 5. Y. Hong, N. M. K. Blackman, N. D. Kopp, A. Sen and D. Velegol, Phys. Rev. Lett., 2007, 99, 178103.

6. M. A. C. Stuart, W. T. S. Huck, J. Genzer, M. Müller, C. Ober, M. Stamm, G. B. SukhorukovI, I. Szleifer, V. V. Tsukruk, M. Urban, F. Winnik, S. Zauscher, I. Luzinov and S. Minko, Nat. Mater., 2010, 9, 101.

7. R. Yoshida, Curr. Org. Chem., 2005, 9, 1617.

8. R. Yoshida, Colloid Polym Sci, 2011, 289, 475.

9. P. Kim, L. D. Zarzar, X. He, A. Grinthal and J. Aizenberg, Curr. Opin. Solid St. M, 2011, 15, 236.

65 10. P. Kim, L. D. Zarzar, X. Zhao, A. Sidorenko and J. Aizenberg, Soft Matter, 2010, 6, 750 .

11. L. D. Zarzar, P. Kim and J. Aizenberg, Adv. Mater., 2011, 23, 1442.

12. A. Sidorenko, T. Krupenkin, A. Taylor, P. Fratzl and J. Aizenberg, Science, 2007, 315, 487.

70 13. A. Sidorenko, T. Krupenkin and J. Aizenberg, J. Mater. Chem., 2008 18, 3841 .

14. L. D. Zarzar, P. Kim, M. Kolle, C. J. Brinker, J. Aizenberg and B. Kaehr, Angew. Chem. Int. Edit., 2011, 50, 9356.

15. W. Hong, X. Zhao and Z. Suo, J. Appl. Phys., 2008, 104, 084905.

75 16. W. Hong, Z. Liu and Z. Suo, Int. J. Solids Struct., 2009, 46, 3282.

17. R. Marcombe, S. Cai, W. Hong, X. Zhao, Y. Lapusta and Z. Suo, Soft Matter, 2010, 6, 784 . 